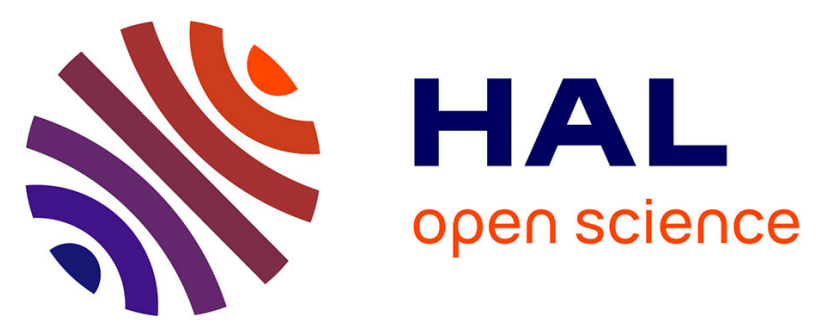

\title{
Effect of frozen turbulence assumption on the local blades vibration on the choke flutter instability in transonic UHBR fan
}

\author{
Pierre Duquesne, Stéphane Aubert, Quentin Rendu, Pascal Ferrand
}

\section{To cite this version:}

Pierre Duquesne, Stéphane Aubert, Quentin Rendu, Pascal Ferrand. Effect of frozen turbulence assumption on the local blades vibration on the choke flutter instability in transonic UHBR fan. IUTAM Symposium on Critical flow dynamics involving moving/deformable structures with design applications, IUTAM, Jun 2018, Santorin, Greece. hal-02146259

\section{HAL Id: hal-02146259 \\ https://hal.science/hal-02146259}

Submitted on 3 Jun 2019

HAL is a multi-disciplinary open access archive for the deposit and dissemination of scientific research documents, whether they are published or not. The documents may come from teaching and research institutions in France or abroad, or from public or private research centers.
L'archive ouverte pluridisciplinaire HAL, est destinée au dépôt et à la diffusion de documents scientifiques de niveau recherche, publiés ou non, émanant des établissements d'enseignement et de recherche français ou étrangers, des laboratoires publics ou privés. 


\title{
EFFECT OF FROZEN TURBULENCE ASSUMPTION ON THE LOCAL BLADES VIBRATION ON THE CHOKE FLUTTER INSTABILITY IN TRANSONIC UHBR FAN
}

\author{
Pierre DUQUESNE ${ }^{1}$, Stéphane AUBERT ${ }^{1}$, Quentin RENDU ${ }^{1}$, Pascal FERRAND ${ }^{1}$ \\ ${ }^{1}$ Université de Lyon, ECL, LMFA UMR CNRS 5509, \\ 36 av. Guy de Collongue 69134 Écully, France
}

\begin{abstract}
.
The choke flutter can lead to the failure of fan or compressor blade in turbojet engines. Choke flutter appears when a strong shock-wave chokes the blade to blade channel. In UHBR fan, choke flutter appears at part speed regimes and at low or negative incidence. In this paper, the choke flutter instability is analyzed based on the resolution of time-linearized Reynolds-Averaged NavierStokes on a 2D blade to blade extraction of an Ultra High Bypass Ratio (UHBR) fan named ECL5v1. An analytical motion of rotation of the airfoil around its leading edge, without the deformation of the blade surface is imposed to a typical choke flutter fan operating condition. For a selected Inter Blade Phase Angle $\left(\mathrm{IBPA}=90^{\circ}\right)$ and selected reduced frequency $(0.15)$, choke flutter instability happens. The blade vibration is decomposed in a large set of subsection to determine the main local flutter sources. This paper aims to analyze the effect of the turbulence modelling on the position of the main local flutter sources and on the work exchange along the blade. Two cases are considered, with the frozen turbulence assumption or with the linerization of the turbulence model. The results locate the main local flutter sources at the same position, with the same work exchange direction but with a modulation of the work exchange magnitude. Amplification or restriction of the magnitude seems to be linked to the steady flow phenomena around both the excitation source and the receptor.
\end{abstract}

Key words: frozen turbulence, choke flutter, L-URANS, UHBR Fan, transonic flow.

\section{Introduction}

The choke flutter can lead to the failure of fan or compressor blade in turbojet engines. Choke flutter appears when a strong shock-wave chokes the blade to blade channel. In UHBR fan, choke flutter appears at part speed regimes (typically 80 $\mathrm{Nn}$ ) and at low or negative incidence (high mass flow, low total pressure ratio). The steady flow is subsonic upstream and downstream of the blade row and supersonic in the blade to blade channel. A strong shock-wave chokes the channel from the suction side to the pressure side.

This paper is the continuity of previous works on a specific case of UHBR fan named ECL5v1. The choke flutter instability is analyzed based on the resolution of time-linearized Reynolds-Averaged Navier-Stokes on a 2D blade to blade extraction. An analytical motion of rotation of the airfoil around its leading edge, without 
the deformation of the blade surface (i.e. a rigid body motion), is imposed to a typical choke flutter fan operating condition. For a selected Inter Blade Phase Angle $\left(\operatorname{IBPA}=90^{\circ}\right)$ and selected reduced frequency $(0.15)$, choke flutter instability happens [4]. In [1], for the same test-case, the blade vibration is decomposed in a large set of subsection to determine the main local flutter sources. The analysis reveals few zones of important work exchange. The exchange induce by the motion of two side by side small zones near the trailing edge on the pressure side lead the overall stability. The direction of the work exchange is different for each zone depending on the phase. The suspected phenomenon, the same in both cases, is the amplification of backward pressure waves at the shock wave. Another couple of sources located on each side of the shock-wave on the suction side has a significant contribution to the overall work exchange. Vibration of these points induce a supplementary motion of the separation bubble and the shock wave.

In the previous works the $k-\omega$ turbulence model is linearized, the main goal of this paper is to apply the same methodology on a set of calculation with the frozen turbulence assumption and compare these results and the results obtain without this assumption.

\section{Numerical methods}

\subsection{Steady RANS solver}

The compressible RANS solver Turb'Flow is used in this work to compute the 2D steady flow in a $90 \%$ height blade to blade channel. This solver relies on vertex centred finite volume method on multi-block structured grids [8].

Convective fluxes are obtained through upwind scheme of Roe [6] with Monotonic Upstream-centred Scheme for Conservative Laws (MUSCL) interpolation of third order [9]. The interpolation order is reduced in strong gradient zones according to Harmonic Cubic Upwind Interpolation (H-CUI) limiter. Diffusive fluxes are obtained through central interpolation of conservative variables. The pseudo time discretisation relies on backward Euler with $\mathrm{CFL}=20$ and local time step to speed up the convergence. The linear problem arising from the implicit method is solved through GMRES iterative method [7].

The flow is considered fully turbulent and the $k-\omega$ turbulence model of Wilcox [11] has been used. At the wall $\omega$ value is extrapolated to be assumed infinite.

\subsection{Time-linearized URANS solver}

The Linearised RANS (LRANS) solver Turb'Lin is used to compute the harmonic flow around the steady state. This solver has been previously validated on transonic separated flows $[3,5]$. The solution is obtained in the frequency domain by solving the linear system. Spatial discretisation relies on Jameson et al. [2] centred scheme with linearised pressure sensor.

\subsection{Aeroelasticity}

The complex amplitude of displacement $\widetilde{\delta \mathbf{x}}$ and velocity $\widetilde{\mathbf{V}}$ is imposed at each node of the blade mesh to model the blades oscillation. The steady position of the blade 


\section{P. DUQUESNE ET AL.}

is chosen as the phase origin. This yields

$$
\Re(\widetilde{\delta \mathbf{x}})=0 \quad ; \quad \Im(\widetilde{\mathbf{V}})=0
$$

The interblade phase angle (IBPA) $\sigma$ is modelled through quasi-periodic boundary conditions in azimuthal direction

$$
\widetilde{\boldsymbol{q}}\left(x_{b}+g\right)=\widetilde{\boldsymbol{q}}\left(x_{b}\right) e^{j \sigma}
$$

where $\widetilde{q}$ is the complex amplitude of conservative variable fluctuations, $x_{b}$ the domain boundary and $g$ the interblade pitch.

The work $\mathcal{W}$ extracted by the flow to the structure is written according to the convention of Verdon [10].

$$
\mathcal{W}=\int_{0}^{T}[-\widetilde{P s}(\mathbf{x}, t) * \mathbf{S}(\mathbf{x}, t)]^{t} \cdot \widetilde{\mathbf{V}}(\mathbf{x}, t) \mathrm{d} t
$$

where $\widetilde{P s}$ is the instantaneous static pressure, $\mathbf{S}$ the vector associated to the instantaneous surface, oriented towards the structure, and $\widetilde{\mathbf{V}}$ the instantaneous velocity vector associated to the blade displacement.

In frequency domain, neglecting second order terms, the only contribution to the unsteady work is, for a rigid body motion,

$$
\Re\left({ }^{1} \widetilde{P s}\right) \mathbf{S} \cdot \Re\left({ }^{1} \widetilde{\mathbf{V}}\right)
$$

where ${ }^{1} \widetilde{P s}$ and ${ }^{1} \widetilde{\mathbf{V}}$ are the complex amplitude of first harmonic of static pressure and velocity vector, respectively. Thus only the real part of fluctuating static pressure contributes to the stability of the fluid-structure interaction.

The damping coefficient is then obtained by the integral of the extracted work normalized by the maximal vibrating kinetic energy along the blade surface

$$
\zeta=\frac{1}{4 \pi} \frac{\iint_{\Omega} \mathcal{W} \mathrm{d} \Omega}{\mathcal{U}}
$$

where $\Omega$ is the fluid-structure contact interface and $\mathcal{U}$ the maximal vibrating kinetic energy of the blade given by

$$
\mathcal{U}=\frac{1}{2} \rho \omega S_{B}
$$

where $\rho$ is the blade material density, $\omega$ the vibration pulsation and $S_{B}$ the total blade surface.

Superposition principle induced by the linearisation of RANS equation leads to the equality between the unsteady flow generated by the vibration of the whole blade and the vibration of each surface mesh node. The blade vibration can thus be decomposed in an arbitrary number of zones $N$ and the global damping coefficient can be computed by the sum of the damping coefficient associated to each vibration. Formally,

$$
\zeta=\sum_{i}^{N} \zeta_{i} ; \quad \zeta_{i}=\frac{1}{4 \pi \mathcal{U}} \iint_{\Omega} \Re\left({ }^{1} \widetilde{P s_{i}}\right) \mathbf{S} \cdot \Re\left({ }^{1} \widetilde{\mathbf{V}}\right) \mathrm{d} \Omega
$$

where $\widetilde{P s_{i}}$ represents the pressure fluctuations generated by the motion of zone $i$. To avoid even-odd decoupling, the blade is decomposed into pairs of adjacent mesh nodes. Each computation consists in the vibration of two adjacent nodes: first 
calculation is computed with the motion of the first and second mesh nodes, next calculation by the motion of the third and fourth nodes, etc. The equality of the global damping coefficient, and its repartition along the chord, for the entire blade vibration and the result of the sum of the motion of each node has been checked. This decomposition strategy has some subtleties: the distance between adjacent nodes is not constant, to compare the contribution of each nodes association the extracted work need to be normalized by the length between the two points. The set of calculations includes 828 L-URANS calculations (half with the calculated turbulence in 424 positions and half with the frozen turbulence assumption at the same positions). All these calculations are based on the same $2 \mathrm{D}$ steady state calculation.

\subsection{Frozen turbulence assumption}

With the frozen turbulence assumption, the turbulence variables are fixed at the time of their steady values, this assumption means that the characteristic time of the turbulence evolution is very slow comparatively to the blade vibration characteristic time (here $300 \mathrm{~Hz}$ ). In opposition, without the frozen turbulence assumption, the turbulence model is also linearized and turbulence variables are calculated in each time step. Physically, that corresponds to consider that the turbulence reacts without delay to the fluctuation of the flow. In previous papers, because of the separated flows the turbulence model has been calculated $[1,3,5]$.

\section{Studied configuration}

\subsection{UHBR fan}

The chosen test case is the Ultra High Bypass Ratio (UHBR) fan ECL5v1. The ECL5 design goals are to generate selected aeroelastic and aerodynamic instabilities, including the choke flutter at part-speed regime, and remains representative of future transonic UHBR fan. The operating range of the ECL5v1 fan, issues from numerical simulations, is plotted in fig. 1 for three different rotational speeds (nominal speed $\mathrm{Nn}=10450 \mathrm{rpm})$.

The energetic method allows to decompose 3D blade in a sum of $2 \mathrm{D}$ airfoils. Most of the extracted work is generated close to the tip due to high levels of both blade velocity and pressure fluctuations. Therefore a 2D blade to blade channel mesh has thus been extracted at $90 \%$ of ECL5v1 height to run the aeroelastic study. At this height the blade surface shows thin, highly staggered blades with low camber, which is typical of transonic fan tip airfoils.

Choke flutter is associated with negative incidence and strong shock-wave choking the interblade channel. It appears for part-speed regime, typically around $80 \%$ of the nominal rotational speed. For the aeroelastic study, the operating point showing the highest massflow on $80 \mathrm{Nn}$ speed characteristic line is thus chosen (in blue in fig. 1). 

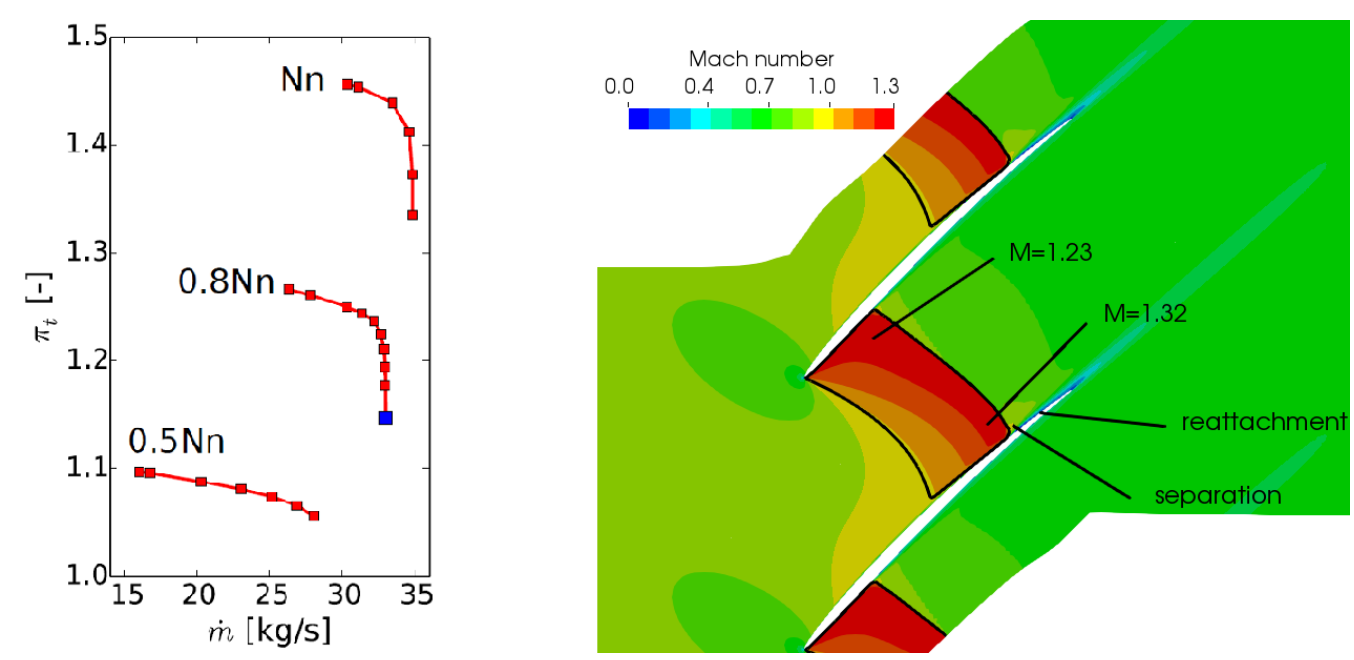

Figure 1: Left: operating range of ECL5v1 - choked operating point in blue. Right: steady relative Mach number for choked flow.

\subsection{Steady flow}

The mesh used for both steady and unsteady computations has been obtained through a convergence study. It consists in 106007 points with $y^{+}<1$ for the first layer of cells close to the blade surface. Total pressure, total temperature and azimuthal velocity are imposed at the upstream boundary and the static pressure at downstream boundary. The boundary conditions of the 2D-steady flow calculation are set to preserve the shock-wave position from the 3D calculation. The steady relative Mach number associated with the choked flow is plotted in fig. 1 .

Looking at the leading edge zone, negative incidence can be seen as well as a supersonic region choking the interblade channel and terminated by a strong shock-wave. On the pressure side, the maximal Mach number is 1.23 and the boundary layer is attached to the blade downstream of the shock-wave. On the suction side, the Mach number reaches 1.32 which leads to the separation of the boundary layer downstream of the shock-wave. The separation is closed and the reattachment point is located $8.3 \%$ of chord downstream of the separation point.

\subsection{Modeshape}

In this study, the chosen mode shape consists in a rotation of the airfoil around its leading edge without the deformation of the blade surface (i.e. a rigid body motion). This mode shape is representative of the first 3D torsion mode of the blade where the transonic flutter is observed. Motion of adjacent blades can present a phase shift called interblade phase angle or IBPA (frequency and mode shape remain identical between blades). The IBPA is by convention positive when the wave propagates in the same direction as the rotor speed and negative otherwise. The reduced frequency, for turbomachinery aeroelastic study, represents the ratio between the time of flight of a fluid particle along the chord and the time of a vibration period. In this work based on previous study, The IBPA is set at $90^{\circ}$ and the reduced frequency is low at 0.15 . The damping coefficient obtained is negative, in the paper convention the work exchange is from the fluid to the blade so this case presents a choke flutter 
instability.

A sketch of three adjacent blades position during the vibration cycle is plotted in fig. 2. For each blade, colours correspond to different instants $(-\mathrm{T} / 4, \mathrm{~T}, \mathrm{~T} / 4)$. Vibration amplitude and interblade distant are modified for illustration purpose. The effective solidity (spacing/chord ratio) is 1.37. The out of phase blades vibration induces different passage section for adjacent interblade channel (see the same instant for the two channel in fig. 2). This area fluctuation leads to strong velocity fluctuations.

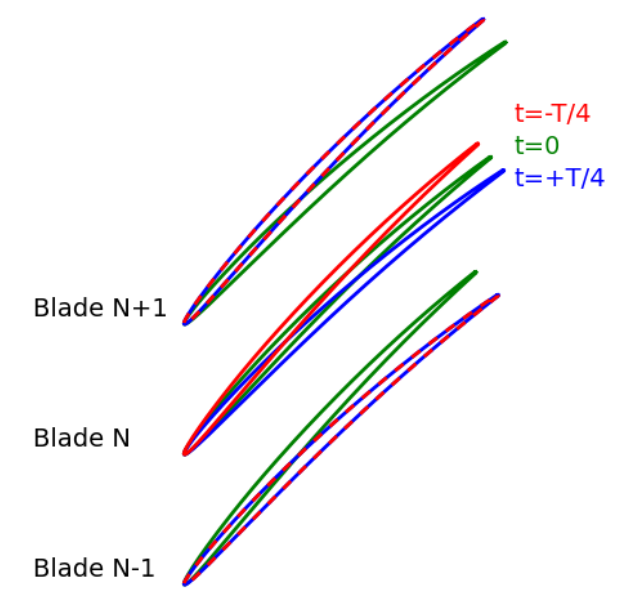

Figure 2: Sketch of the vibration of three adjacent blades at three different instants, airfoil colours show the different instants: $-\mathrm{T} / 4, \mathrm{~T}, \mathrm{~T} / 4$. Vibration amplitude and interblade distant are modified for illustration purpose.

\section{Effect of frozen turbulence assumption}

\subsection{Effect on the total damping coefficient}

The total damping coefficient $\zeta$ (calculate with eq. 5 ) is negative, which corresponds to the blade vibration amplification (flutter case), independently of the frozen turbulence assumption use. The damping coefficient magnitude is smaller, but in the same order of magnitude, for the frozen turbulence case. The damping coefficient difference between both cases is similar to the difference when another turbulence model is chosen, in this sense the frozen turbulence assumption has a restricted effect on the prediction of the damping coefficient.

The work exchange, normalized by the maximal vibrating kinetic energy and element surface, along the chord is plotted in fig. 3 for the calculated turbulence case (blue line) and for the frozen turbulence case (dashed red). The smaller damping coefficient for the frozen turbulence case is induced by a smaller work exchange downstream to the shock-wave. The work exchange reduction is more important on the suction side in particular in the flow separation bubble zone. This result suggests that the effect of the frozen turbulence assumption is concentrated on the flow separation bubble that is consistent with the importance of viscous effects in this type of secondary flow. The flow downstream the shock wave is indirectly impacted 


\section{P. DUQUESNE ET AL.}

by the effect on the flow separation bubble and the flow upstream is not affected protected by the strong shock-wave which chokes the interblade channel.

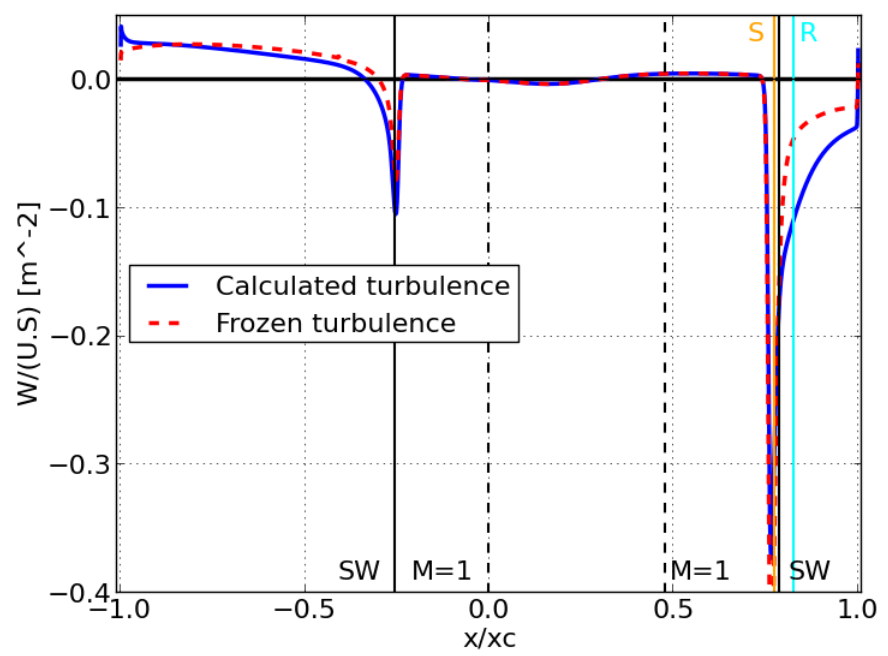

Figure 3: Extracted normalized work along blade chord for calculated or frozen turbulence (leading edge at $\mathrm{x} / \mathrm{xc}=0$, pressure side: $\mathrm{x} / \mathrm{xc}<0$, suction side: $\mathrm{x} / \mathrm{xc}>0$ ). Separation, reattachment, sonic line and shock-wave positions are represented by vertical lines noted $\mathrm{S}, \mathrm{R}$, $\mathrm{M}=1$ and $\mathrm{SW}$ respectively.

\subsection{Effect on main flutter source}

To determine the main flutter sources, the sum of the work along the chord is performed for the individual motion of each point. Results for both sets of calculations are represented in fig. 4, the case with calculated turbulence on the left and with the frozen turbulence assumption in the middle. The right side of fig. 4 shows the enlargement of the trailing edge in both cases. The colours correspond to the work around the airfoil for the motion of the segment at this position. Here, the work is normalized by the length of the moving point to determine main flutter sources without the effect of the segment length. Colour scale has been restricted for presentation purpose due to local high values near the trailing edge. In fig. 4, the supersonic zone is delimited by black lines and the position of the separation point and reattachment point are reported by $(S)$ and $(R)$ respectively.

The global repartition pattern of the flutter sources is similar to both sets of calculations: the overall stability is induced by few localized excitation source with high intensity near the trailing edge. The stabilizing or destabilizing aspect of main local sources have the same direction in both cases. Four particular zones have been selected because of their high contribution to stability in a restricted area. In addition to having the same stabilizing or destabilizing behaviour, main flutter sources with or without calculated turbulence have the same size to one mesh point. These zones are listed in table 1 and reported in fig. 4 with a reference letter (A,B,C,D). In both set of calculation zones ares the same. The A-zone is located on the pressure side near (but not at) the trailing edge. The vibration of this small zone, less than 

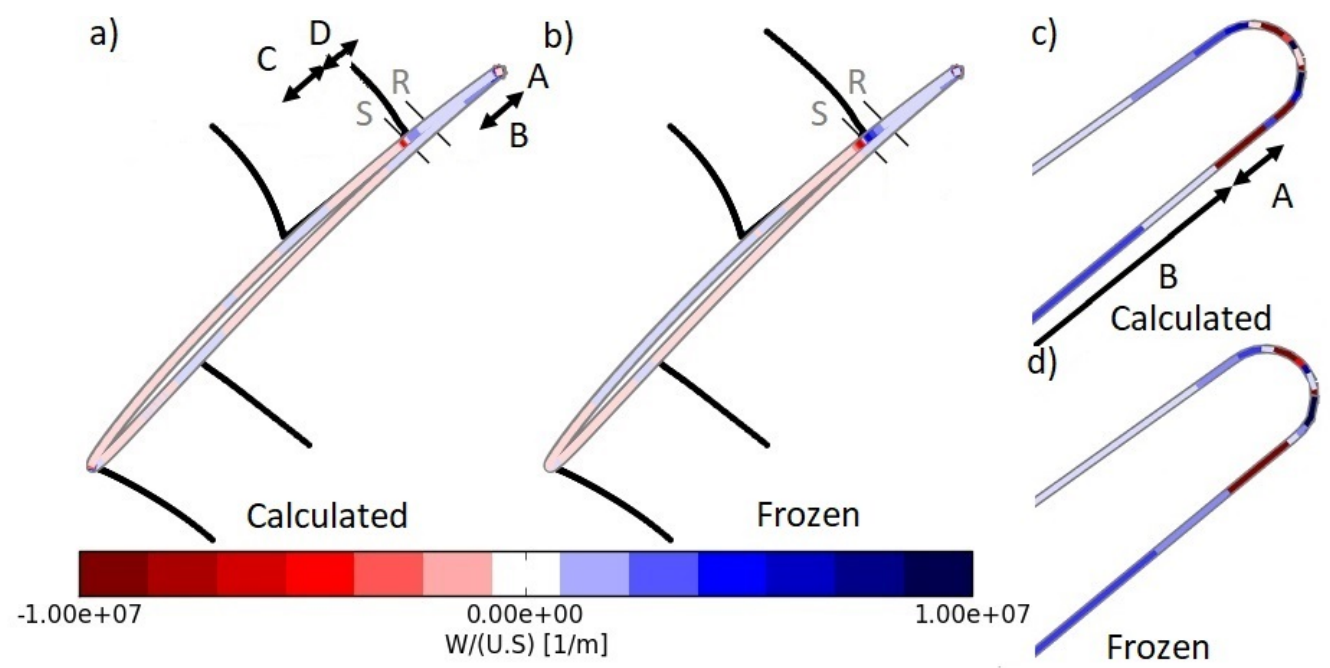

Figure 4: Normalized work on the airfoil, sonic line is in black, $(\mathrm{S})$ and $(\mathrm{R})$ are respectively separation and reattachment points, selected zones are referred by A,B,C,D. Left airfoil represents results for calculated turbulence, middle figure for frozen turbulence assumption and the trailing edge enlargement at right.

$0.1 \%$ of the total airfoil length, induces the largest part of the negative (destabilizing) work. The vibration of the zone just upstream the A-zone on the pressure side (B-zone) induces a large part of the positive (stabilizing) work. Even if B-zone is larger than the A-zone, it is kept small (4.2\% of the total airfoil length). The stabilizing work induced by the motion of the B-zone cannot compensate for the large amount of destabilizing work induced by the motion of the A-zone : the cumulative work of A and B-zones is negative (destabilizing). On the suction side, most of the contribution on the stability is again induced by the motion of points located near the trailing edge. The C-zone is just upstream the shock-wave and the D-zone downstream. D-zone corresponds to the flow separation bubble (between the separation and the reattachment point). The separation point is the border between $\mathrm{C}$ and $\mathrm{D}$ zones and correspond to an inversion of the work exchange. $\mathrm{C}$ zone induces negative (destabilizing) instead of D-zone that induces positive (stabilizing) work. The proportion to the contribution to the stabilizing work and the destabilizing work induce by both zones are similar.

The frozen turbulence assumption impact magnitude of all flutter sources but in different ways. The frozen turbulence assumption tends to predict smaller work exchange for zones $\mathrm{A}$ and $\mathrm{B}$ and a larger work exchange for zones $\mathrm{C}$ and $\mathrm{D}$. For example, the contribution of zone $\mathrm{C}$ on the total destabilizing work is twice when frozen turbulence assumption is used (from 17 to 34\%). The same effect can be observed for zone D and in the same time contribution of zones A and B decrease. Despite that, the pressure side flutter sources always lead the global stability. The balance between zone A and B is smaller when using frozen turbulence assumption but always negative (destabilizing) and larger than other sources. In comparison, the couple zone $\mathrm{C}$ and zone $\mathrm{D}$ tend to cancel their effect mutually in both cases. Due to this result, the next analysis is focused on the work exchange induced by the motion of a point of $\mathrm{A}$ and $\mathrm{B}$ zone only. 


\section{P. DUQUESNE ET AL.}

Table 1: Selected region name, work (-:destabilizing, +:stabilizing) for calculated and frozen turbulence, the percentage in bracket is the work contribution induce by the segment motion in the destabilizing part of the work (or stabilizing case dependent) and the zone length versus the airfoil length.

\begin{tabular}{cccc}
\hline Zone name & $\begin{array}{c}\text { Calculated } \\
\mathcal{W} / \mathcal{U} .10^{-3}\end{array}$ & $\begin{array}{c}\text { Frozen } \\
\mathcal{W} / \mathcal{U} .10^{-3}\end{array}$ & $\begin{array}{c}\text { Blade } \\
\text { length }\end{array}$ \\
\hline$A$ & $-11.5(56 \%)$ & $-7.9(44 \%)$ & $0.1 \%$ \\
$B$ & $+8.5(44 \%)$ & $+5.7(32 \%)$ & $5 \%$ \\
$C$ & $-3.6(17 \%)$ & $-6.1(34 \%)$ & $4 \%$ \\
$D$ & $+3.1(16 \%)$ & $+6.1(34 \%)$ & $2 \%$ \\
Airfoil & -1.4 & -0.3 & \\
Sum $\mathcal{W} / \mathcal{U}<0$ & -20.6 & -18 & \\
Sum $\mathcal{W} / \mathcal{U}>0$ & +19.2 & +17.7 &
\end{tabular}

\subsection{Effect on the stability induced by pressure side zones}

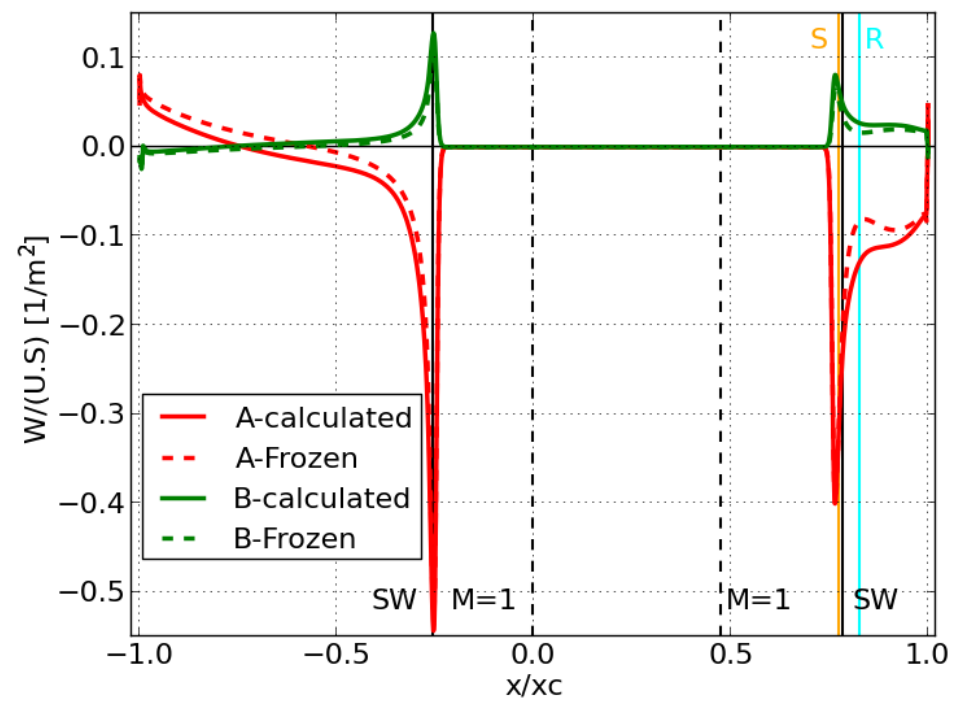

Figure 5: Normalised extracted work along blade chord products by the motion of a point in zone $\mathrm{A}$ and $\mathrm{B}$ (leading edge at $\mathrm{x} / \mathrm{xc}=0$, pressure side: $\mathrm{x} / \mathrm{xc}<0$, suction side: $\mathrm{x} / \mathrm{xc}>0$ ). Separation, reattachment, sonic line and shock-wave positions are represented by vertical lines noted S, R, M=1 and SW respectively. Solid and dashed line for calculated and frozen turbulence respectively.

Fig. 5 presents the extracted work along the blade chord for the motion of the most destabilizing point from A-zone (in red) and for the motion of the most stabilizing point from B-zone (in green). Solid line and dashed lines represent results for calculated turbulence and frozen turbulence cases respectively. The two selected points are close together and on the pressure side downstream the shock-wave near the trailing edge. As is postulated in [1], backward pressure waves generated by the motion of a point in zone A or B travel upstream in the blade to blade channel up to the shock-wave. When the backward pressure waves reach the shock-wave their amplitude increased. The amplification of the pressure fluctuation induced prod- 
ucts more work exchange. Results for frozen turbulence assumption shows a more stabilizing behavior downstream the shock-wave in particular in the flow separation bubble.

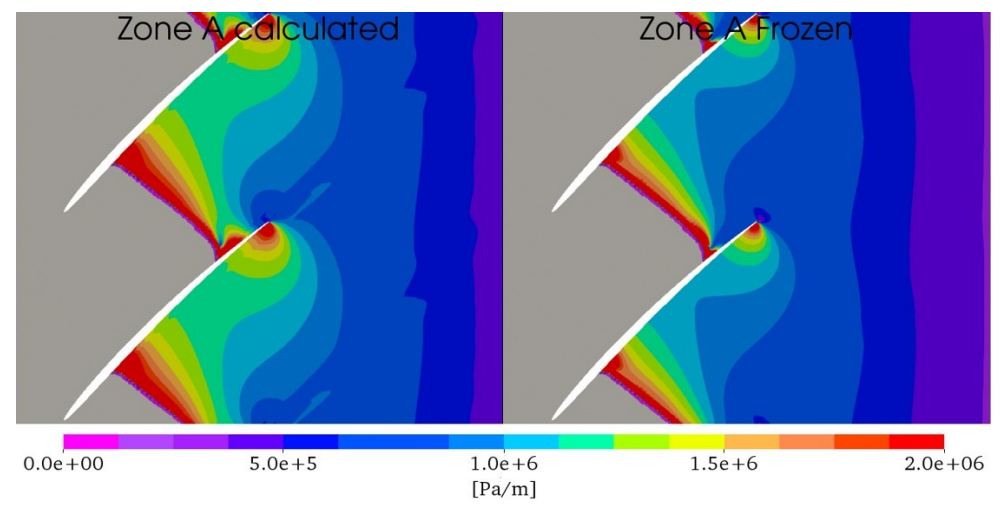

Figure 6: Pressure fluctuation modulus induces by the motion of the most destabilizing point in A-zone.Calculated and frozen turbulence respectively at left and right

The pressure fluctuation modulus for the motion of a point in zone A with or without frozen turbulence assumption are presented in fig. 6 (the pressure fluctuation modulus for a point in zone B have the same pattern with a smaller amplitude). At the excitation sources, the pressure fluctuation produced by the motion of the segment is larger with the frozen turbulence assumption, but as shown in fig. 6, pressure fluctuation is smaller in the neighbourhood of the source. The frozen turbulence tends to reduce the propagation of the pressure waves. Also, the frozen turbulence reduces the interaction of the pressure wave with the flow separation bubble and leads to smaller pressure fluctuations in this region. This observation tends to confirm the hypothesis that the higher pressure fluctuation gradient on the suction side is explained by the interaction with the flow separation bubble as formulated in [1]. The frozen turbulence assumption seems leading to smaller pressure fluctuations where viscous effects are important.

The direction of the work exchange seems to be linked to the phase of the excitation source. The phase mapping of the motion of a point in A zone (stabilizing) and in B zones (destabilizing) have similar pattern but with a $180^{\circ}$ phase shift. The frozen turbulence assumption has no effect on the phase shift. The representation of turbulence plays here a minor part. The direction of the work exchange is not sensitive to the viscous effect and the mechanism of amplification of the pressure wave at the shock-wave appears majority like a potential effect.

In fig. 7 the root of the turbulent kinetic energy fluctuation modulus and the velocity fluctuation modulus, both normalized by the averaged velocity norm, are represented. The map of the turbulent kinetic energy fluctuation modulus is not represented for the frozen turbulence, by definition this one is null everywhere. Due to the small displacement assumption in the linearized calculation, the fluctuation values don't correspond to the real fluctuation but are scaled for a unitary displacement.

As shown in fig. 7, the velocity fluctuation is more prominent at the shock-wave and in the flow separation bubble in both cases. The velocity fluctuation at the shock 


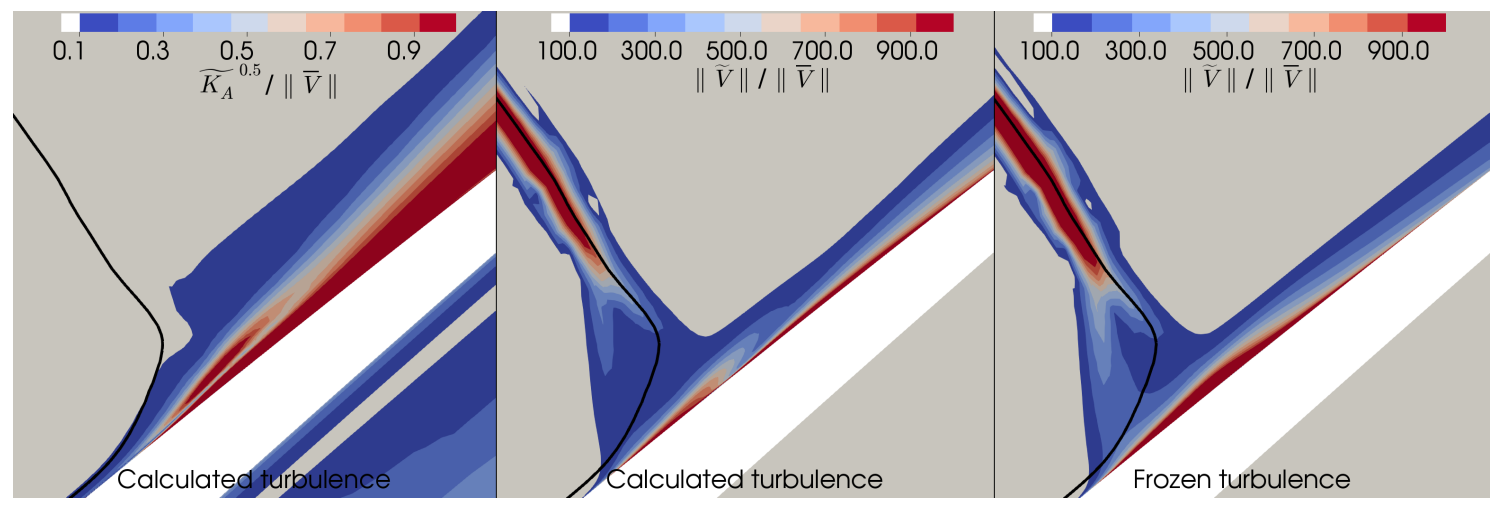

Figure 7: Squared normalized turbulent kinetic energy fluctuation modulus induces by the motion of the most destabilizing point in A-zone for calculated turbulence at left. Normalized velocity fluctuation modulus induces by the same point for calculated and frozen turbulence respectively in the middle and at right. Black lines represent the steady shock-wave.

wave is relatively similar with or without frozen turbulence assumption. Contrary to inside and near the flow separation bubble, the velocity fluctuation is more important and more constant in the case of the frozen turbulence. In the same positions, the turbulent kinetic energy fluctuates when the turbulence is calculated at each time step. In the case of the frozen turbulence assumption, the fluctuation of the total energy can only be redirected to the term of pressure fluctuation and velocity fluctuation. In the case of the calculated turbulence, the term of the turbulence can absorb a part of the energy fluctuation. Therefore the turbulence (when calculated at each time) acts like a damper in the boundary layer and limit the velocity fluctuation. Even if the turbulence fluctuation is three orders of magnitude smaller than velocity fluctuation they have a non-negligible effect on the work exchange induced by the vibration of the A-zone.

\section{Conclusions}

The choke flutter in an Ultra High Bypass Ratio fan is analyzed using a timelinearized Reynolds-Averaged Navier-Stokes equation solver on a 2D blade to blade extraction at $90 \%$ height. Two cases for the $k-\omega$ turbulence model are considered: with the turbulence quantities calculated at each time step or using the frozen turbulence assumption. A vibrating decomposition method based on the superposition principle is performed on each surface mesh node to identify the main sources of the work exchange between the flow and the blade.

In both cases, with and without frozen turbulence assumption, the damping coefficient and the position of most intense destabilizing sources and stabilizing sources are similar. The overall stability is leading by two couples of stabilizing/destabilizing sources. The first couple is downstream of the shock-wave near the trailing edge on pressure side. The vibration of this zone induces backward travelling pressure waves, which propagates upstream up to the shock-wave. The next couple is on the suction side in the neighborhood of the interaction between the shock-wave and the flow 
separation. The destabilizing source is just upstream the shock-wave and the stabilizing source correspond to the flow separation bubble. For both couples of sources, the stability direction is linked to the phase, stabilizing sources and destabilizing sources have a phase shift of $180^{\circ}$.

The frozen turbulence assumption plays on the amplitude of these two couples of sources. With the frozen turbulence assumption, the main source on the pressure side has a smaller work exchange and, in opposition, the work exchange is more intense for the main source on the suction side. The frozen turbulence has more effect where the viscous effects are important in the flow dynamics (i.e. in the flow separation bubble). Analysis of turbulent kinetic energy fluctuations and velocity fluctuations of the most destabilizing zone (A-zone) reveals that the acts like a damper in the boundary layer and limit the velocity fluctuation when turbulence is calculated at each time step. The small proportion of total energy absorbed by the turbulence has a non-negligible impact on the work exchange. This test-case shows an example of the importance to calculate the turbulence at each time step, in particular when flow phenomena are driven by viscous effects.

\section{References}

[1] P. Duquesne, Q. Rendu, P. Ferrand, and S. Aubert. Local contribution of blades vibration on the choke flutter instability in transonic UHBR fan. In 53rd 3AF International Conference on Applied Aerodynamics, Salon de Provence, France, 2018.

[2] A. Jameson, W. Schmidt, and E. Turkel. Numerical solution of the Euler equations by finite volume methods using Runge Kutta time stepping schemes. In 14th Fluid and Plasma Dynamics Conference, Palo Alto, USA, 1981. American Institute of Aeronautics and Astronautics.

[3] M. Philit, P. Ferrand, S Labit, J.-C. Chassaing, S. Aubert, and T. Fransson. Derivated turbulence model to predict harmonic loads in transonic separated flows over a bump. In 28th International Congress of Aeronautical Sciences, Brisbane, Australia, 2012.

[4] Q. Rendu, S. Aubert, and P. Ferrand. Influence of reduced frequency on choke flutter instability in transonic UHBR fan. In International Forum on Aeroelasticity and Structural Dynamics, Como, Italy, June 2017.

[5] Q. Rendu, M. Philit, S. Labit, J.-C. Chassaing, R. Yannick, S. Aubert, and P. Ferrand. Time-linearized and harmonic balance Navier-Stokes computations of a transonic flow over an oscillating bump. In 11th International Symposium on Unsteady Aerodynamics, Aeroacoustics and Aeroelasticity of Turbomachines., Stockholm, Sweden, September 2015.

[6] P.L. Roe. Approximate Riemann solvers, parameter vectors, and difference schemes. Journal of Computational Physics, 43(2):357-372, October 1981.

[7] Y. Saad and M. Schultz. GMRES: A generalized minimal residual algorithm for solving nonsymmetric linear systems. SIAM J. Sci. and Stat. Comput., 7(3):856-869, July 1986.

[8] L. Smati, S. Aubert, P. Ferrand, and F. Massão. Comparison of numerical schemes to investigate blade flutter. In Unsteady Aerodynamics and Aeroelasticity of Turbomachines, pages 749-763. Springer, Dordrecht, 1998.

[9] B. Van Leer. Towards the ultimate conservative difference scheme. V. A second-order sequel to Godunov's method. Journal of Computational Physics, 32(1):101-136, July 1979.

[10] J. Verdon. Linearized unsteady aerodynamic theory. In AGARD Manuel on Aeroelasticity in Axial-Flow Turbomachines., volume Volume 1. Unsteady Turbomachinery Aerodynamics. Advisory Group for Aerospace Research and Development, Neuilly-sur-Seine (France), 1987.

[11] D.C. Wilcox. Reassessment of the scale-determining equation for advanced turbulence models. AIAA Journal, 26(11):1299-1310, 1988. 